\title{
Plasmons in realistic graphene/hexagonal boron nitride moiré patterns
}

\author{
Andrea Tomadin, ${ }^{1,2, *}$ Marco Polini, ${ }^{2}$ and Jeil $\mathrm{Jung}^{3, \dagger}$ \\ ${ }^{1}$ Department of Physics, Lancaster University, Lancaster LA1 $4 Y B$, United Kingdom \\ ${ }^{2}$ Istituto Italiano di Tecnologia, Graphene Labs, Via Morego 30, 16163 Genova, Italy \\ ${ }^{3}$ Department of Physics, University of Seoul, Seoul 02504, Korea
}

(Dated: January 5, 2019)

\begin{abstract}
Van der Waals heterostructures employing graphene and hexagonal boron nitride (hBN) crystals have emerged as a promising platform for plasmonics thanks to the tunability of their collective modes with carrier density and record values for plasmonics figures of merit. In this Article we investigate theoretically the role of moiré-pattern superlattices in nearly aligned graphene on hBN by using a continuum-model Hamiltonian derived from $a b$ initio calculations. We calculate the system's energy loss function for a range of chemical potential values that are accessible in gated devices. Our calculations reveal that the electron-hole asymmetry of the moiré bands leads to a remarkable asymmetry of the plasmon dispersion between positive and negative chemical potentials, showcasing the intricate band structure and rich absorption spectrum across the secondary Dirac point gap for the hole bands.
\end{abstract}

\section{INTRODUCTION}

Research on graphene has been actively pursued during the past decade after seminal experiments achieving its isolation via micromechanical cleavage and subsequent transport measurements. ${ }^{1-5}$ Graphene shows semimetallic behavior with linearly dispersing massless Dirac-fermion bands near charge neutrality while hexagonal boron nitride $(\mathrm{hBN})$ is a wide band-gap insulator. Devices based on graphene on hBN substrates ${ }^{6-8}$ have shown a dramatic enhancement of electronic quality compared to $\mathrm{SiO}_{2}$ substrates, due to the atomically smooth surface structure of $\mathrm{hBN}$ and the relatively smaller number of charge trap centers due to dangling bonds. This has allowed the observation of fine Coulombinteraction-driven phenomena such as fractional quantum Hall states, ${ }^{9,10}$ enhancement of the Fermi velocity, ${ }^{11}$ and strong Coulomb drag, ${ }^{12}$ to mention a few examples.

The use of hBN substrates allows to engineer the electronic structure of graphene by introducing moiré superlattice band features and band gaps at the primary and secondary Dirac points. ${ }^{13-19}$ In the limit where the layers are nearly aligned, graphene on $\mathrm{hBN}(\mathrm{G} / \mathrm{hBN})$ displays moiré superlattice patterns with periodicity as large as $\lambda \sim 14 \mathrm{~nm}$, which allows to access moiré-pattern-induced electronic structure features at carrier densities accessible with conventional electrostatic gating. The presence of a long periodic superlattice structure together with the high quality of the devices has allowed the observation of Hofstadter butterfly physics in the presence of magnetic fields routinely accessible in the laboratory. ${ }^{20,21}$ The moiré periodicity $\lambda$ depends on the relative difference $\epsilon=\left(a_{\mathrm{G}}-a_{\mathrm{BN}}\right) / a_{\mathrm{BN}}$ between graphene's and hBN's lattice constants, $a_{\mathrm{G}}$ and $a_{\mathrm{BN}}$, respectively, and on the relative twist angle $\theta$, through $\lambda \simeq a_{\mathrm{G}} /\left(\theta^{2}+\epsilon^{2}\right)^{1 / 2}$. Because of this dependence, the layer orientation is an additional knob for modifying the electronic structure. The band gaps observed at the primary ${ }^{15,22,23}$ and secondary Dirac points ${ }^{24}$ reflect the effect of moiré strains inducing an average mass term, ${ }^{17,25-27}$ while realistic models for the moiré pattern potentials can capture the secondary Dirac point gaps on the hole side. ${ }^{18,19}$ Fairly accurate moiré patterns can be modeled already in the first harmonic approximation for slowly varying potentials in the basis of the identity and Pauli matrices, whose details determine the character of the moiré superlattice bands such as the electron-hole asymmetry ${ }^{18}$ and the presence of secondary gaps. ${ }^{19}$

A detailed study of the collective behavior induced by long-range Coulomb interactions in $\mathrm{G} / \mathrm{hBN}$ systems is of considerable value for advancing our understanding of 2D-material-based plasmonics. Indeed, it has been recognized early on that graphene and other two-dimensional materials exhibit very interesting optoelectronic properties. $^{28}$ In particular, G/hBN systems have been identified as a promising platform for plasmonic applications, ${ }^{29}$ featuring e.g. electrical tunability of the plasmon dispersion, high compression of electromagnetic radiation, and facile coupling to emitters adjacent to the graphene sheet. Most importantly, it has been shown ${ }^{30}$ that graphene encapsulated between hBN crystals supports plasmon propagation with room-temperature lifetime $\tau_{\mathrm{p}}$ exceeding $500 \mathrm{fs}$, which represents a five-fold enhancement compared to that achieved in the case of $\mathrm{SiO}_{2}{ }^{31}$ or $\mathrm{SiC}^{32}$ substrates. The propagation of plasmons in graphene/hBN systems has also been used to reconstruct the local conductivity, ${ }^{33}$ and thus verify the modification of the electronic structure due to the moiré pattern. More recently, the plasmonic properties of encapsulated graphene have been explored at liquid-nitrogen temperatures, where plasmon lifetimes on the order of 1.600 fs have been measured. ${ }^{34}$

These breakthroughs have fostered a substantial research activity into the optoelectronic properties of the large family of two-dimensional materials, which includes semimetals, semiconductors, and insulators. ${ }^{35,36}$ These materials feature several light-matter hybrid modes, generally referred to as "polaritons," which are supported by the electric polarization of free carriers, excitonic states, or lattice ions. 
Previous studies of the electron energy loss in $\mathrm{G} / \mathrm{hBN}$ moiré patterns, ${ }^{37}$ which neglected gauge fields, demonstrated that the dispersion of plasmonic excitations in graphene sensitively depends on the Hamiltonian details. In this Article we take a step further by exploring plasmons in $\mathrm{G} / \mathrm{hBN}$ in a wide chemical potential range accessible in experiments by using a realistic electronic structure model for $\mathrm{G} / \mathrm{hBN}$ moiré patterns. ${ }^{17,19,38}$ The manuscript is structured as follows. In Sec. II we present the continuum-model effective Hamiltonian for G/hBN, summarizing the framework to calculate a set of realistic parameters for the moiré potential, and we detail the expression for the dielectric and loss functions within the random phase approximation (RPA) ${ }^{39}$ The results of our numerical calculations are presented and discussed in Sec. III. Finally, in Sec. IV we summarize our work and draw our main conclusions.

\section{THE MODEL}

\section{A. Effective Hamiltonian}

The effect of interlayer coupling on graphene's band structure can be modelled through the following Hamiltonian, where we use the notation of Ref. 37, including scalar, mass, and gauge potentials:

$$
\begin{aligned}
\hat{\mathcal{H}}= & v_{\mathrm{F}} \tau_{0} \boldsymbol{\sigma} \cdot \hat{\boldsymbol{p}}+\tau_{0} \sigma_{0} V(\boldsymbol{r})+\tau_{3} \sigma_{3}\left[\Delta_{0}+\Delta(\boldsymbol{r})\right] \\
& +\tau_{3} \boldsymbol{\sigma} \cdot \boldsymbol{A}(\boldsymbol{r}) .
\end{aligned}
$$

The position vector $\boldsymbol{r}$ lies in the two-dimensional (2D) plane where the graphene sheet lies. The Pauli matri- ces $\sigma_{i}$ operate on the pseudospin space spanned by the sublattice sites $A, B ; \tau_{i}$ acts on the space of graphene's principal valleys $K, K^{\prime}$; and $\boldsymbol{\sigma}=\left(\sigma_{1}, \sigma_{2}\right)$ is a vector of Pauli matrices. We parametrize the spatial distribution of the moiré pattern as follows ${ }^{19,40}$

$$
\begin{aligned}
V(\boldsymbol{r}) & =2 C_{0} \Re e\left[e^{i \phi_{0}} f(\boldsymbol{r})\right], \\
\Delta(\boldsymbol{r}) & =2 C_{z} \Re e\left[e^{i \phi_{z}} f(\boldsymbol{r})\right], \\
\boldsymbol{A}(\boldsymbol{r}) & =2 C_{A B} \chi \hat{z} \times \vec{\nabla} \Re e\left[e^{i \phi_{x y}} f(\boldsymbol{r})\right] .
\end{aligned}
$$

Here, $C_{\mu}$ and $\phi_{\mu}$ are numerical constants that we discuss below. The coefficient $\chi$ depends on the twist angle $\theta$ and is given by

$$
\chi=\frac{1+\epsilon-\cos (\theta)}{\sqrt{(1+\epsilon)^{2}+2(1+\epsilon) \cos (\theta)+1}} .
$$

Finally, the complex function $f(\boldsymbol{r})$ is given by

$$
f(\boldsymbol{r})=\sum_{m=1}^{6} e^{i \boldsymbol{G}_{m} \cdot \boldsymbol{r}} \frac{1+(-1)^{m}}{2}
$$

where $\boldsymbol{G}_{m}$ are the six reciprocal lattice vectors of the moiré pattern which are closer to the origin. The Hamiltonian (1) can be rewritten in the more explicit form

$$
\hat{\mathcal{H}}=v_{\mathrm{F}} \tau_{0} \boldsymbol{\sigma} \cdot \hat{\boldsymbol{p}}+\tau_{3} \sigma_{3} \Delta_{0}+\sum_{m=1}^{6} e^{i \boldsymbol{G}_{m} \cdot \boldsymbol{r}} W_{m},
$$

where

$$
W_{m}=\left(\begin{array}{cc}
W_{m, 1} & 0 \\
0 & W_{m,-1}
\end{array}\right), \quad W_{m, \nu}=\left(\begin{array}{cc}
C_{0} e^{i(-1)^{m} \phi_{0}}+\nu C_{z} e^{i(-1)^{m} \phi_{z}} & \nu C_{A B} \chi e^{i\left[-\varphi_{m}+(-1)^{m+1} \phi_{x y}\right]} \\
-\nu C_{A B} \chi e^{i\left[\varphi_{m}+(-1)^{m+1} \phi_{x y}\right]} & C_{0} e^{i(-1)^{m} \phi_{0}}-\nu C_{z} e^{i(-1)^{m} \phi_{z}}
\end{array}\right)
$$

Here, $\varphi_{m}$ is the polar angle of the wave vector $\boldsymbol{G}_{m}$ and the order of the basis vectors in the 4-dimensional sublattice/valley space is $\left\{|A K\rangle,|B K\rangle,\left|B K^{\prime}\right\rangle,-\left|A K^{\prime}\right\rangle\right\}$.

The Hamiltonian for $\mathrm{G} / \mathrm{hBN}$ that we use in this work is based on ab initio calculations for the interlayer coupling, which capture effects beyond the commonly assumed two-center approximation. ${ }^{38}$ In Eq. (2), the magnitude and phase pairs $C_{\mu}, \phi_{\mu}$ are moiré pattern parameters that capture the effective interlayer coupling in the first harmonic approximation. ${ }^{19,38}$ The parameters $C_{\mu}$, $\phi_{\mu}$ were obtained by first calculating the distant hopping terms from carbon to the substrate atoms, for all possible commensurate stacking configurations. Then, the real-space hopping terms were Fourier transformed, to calculate the effective interlayer coupling near the Dirac point, for every stacking configuration. An additional Fourier transform in the reciprocal lattice vectors for the moire patterns led to the $W_{m}$ terms used in Eq. (7) and defined in Eq. (8). The parameters used in our model Hamiltonian are:

$$
\begin{aligned}
& C_{0}=0.01013 \mathrm{eV}, \quad \phi_{0}=26.53^{\circ}, \\
& C_{z}=0.00901 \mathrm{eV}, \quad \phi_{z}=-51.57^{\circ}, \\
& C_{A B}=0.01134 \mathrm{eV}, \quad \phi_{x y}=130.40^{\circ} .
\end{aligned}
$$

The model also needs the following additional parameters:

$$
\begin{aligned}
& \Delta_{0}=0.010 \mathrm{eV}, \quad \epsilon=-0.017, \\
& v_{\mathrm{F}}=1.1 \mathrm{~nm} / \mathrm{fs}, \quad \alpha_{\mathrm{ee}}=1.0,
\end{aligned}
$$

where $\Delta_{0}$ is the magnitude of the average gap that can be introduced by moiré strains, ${ }^{17} v_{\mathrm{F}}$ is the Fermi velocity in $\mathrm{G} / \mathrm{hBN}$, and $\alpha_{\mathrm{ee}}=e^{2} /\left(\bar{\epsilon} \hbar v_{\mathrm{F}}\right)$ is a dimensionless coupling constant measuring the strength of electron-electron interactions, with $e$ the electron's charge and $\bar{\epsilon}$ the average dielectric constant. 
We point out that a different set of Hamiltonian parameters $u_{i}, \tilde{u}_{i}$, based on inversion symmetry considerations and a choice of origin, was also analyzed in the literature. ${ }^{16,41}$ The relation between the sets $u_{i}, \tilde{u}_{i}$ and $C_{\mu}, \phi_{\mu}$ is discussed in detail in Ref. 19.

\section{B. Theory of the dielectric and loss functions for moiré superlattices}

The dielectric function $\epsilon_{\boldsymbol{G}, \boldsymbol{G}^{\prime}}(\boldsymbol{q}, \omega)$ relates the external potential $V_{\text {ext }}(\boldsymbol{G}+\boldsymbol{q}, \omega)$ applied to the electron system and the total potential $V_{\mathrm{sc}}(\boldsymbol{G}+\boldsymbol{q}, \omega)$, which includes the contribution of the displaced carrier's charges (i.e. electronic screening), according to the relation

$$
\sum_{\boldsymbol{G}^{\prime}} \epsilon_{\boldsymbol{G}, \boldsymbol{G}^{\prime}}(\boldsymbol{q}, \omega) V_{\mathrm{sc}}\left(\boldsymbol{G}^{\prime}+\boldsymbol{q}, \omega\right)=V_{\mathrm{ext}}(\boldsymbol{G}+\boldsymbol{q}, \omega) .
$$

Here, $\boldsymbol{G}$ are reciprocal lattice vectors of the moiré pattern superlattice and $\boldsymbol{q}$ is a wave vector in the moiré Brillouin zone (mBZ). Differently from a homogeneous system, the dielectric function is a matrix $\boldsymbol{\epsilon}$ in the reciprocal lattice space ${ }^{39}$ because the wave vector of the external potential is conserved only up to a reciprocal lattice vector of the mBZ.

Within the RPA, the dielectric function is related to the non-interacting density-density polarization function (i.e. the Lindhard function) $\chi_{\boldsymbol{G}, \boldsymbol{G}^{\prime}}^{(0)}(\boldsymbol{q}, \omega)$ by $^{39,42}$

$$
\epsilon_{\boldsymbol{G}, \boldsymbol{G}^{\prime}}(\boldsymbol{q}, \omega)=\delta_{\boldsymbol{G}, \boldsymbol{G}^{\prime}}-v_{\boldsymbol{G}}(\boldsymbol{q}) \chi_{\boldsymbol{G}, \boldsymbol{G}^{\prime}}^{(0)}(\boldsymbol{q}, \omega),
$$

where $v_{\boldsymbol{G}}(\boldsymbol{q})=v(\boldsymbol{q}+\boldsymbol{G})$ with $v(q)=2 \pi e^{2} /(\bar{\epsilon} q)$ is the 2D Fourier transform of the Coulomb potential.

The explicit expression for the Lindhard function is

$$
\begin{aligned}
& \chi_{\boldsymbol{G}, \boldsymbol{G}^{\prime}}^{(0)}(\boldsymbol{q}, \omega)=\frac{2}{L^{2}} \sum_{\boldsymbol{k}, n ; \boldsymbol{k}^{\prime}, n^{\prime} ; \nu} \frac{n_{\mathrm{F}}\left(\varepsilon_{\boldsymbol{k}, n, \nu}\right)-n_{\mathrm{F}}\left(\varepsilon_{\boldsymbol{k}^{\prime}, n^{\prime}, \nu}\right)}{\hbar \omega+\varepsilon_{\boldsymbol{k}, n, \nu}-\varepsilon_{\boldsymbol{k}^{\prime}, n^{\prime}, \nu}+i \eta} \\
& \times \mathcal{M}_{\boldsymbol{k}, n, \nu ; \boldsymbol{k}^{\prime}, n^{\prime}, \nu}(\boldsymbol{q}+\boldsymbol{G}) \mathcal{M}_{\boldsymbol{k}, n, \nu ; \boldsymbol{k}^{\prime}, n^{\prime}, \nu}^{\dagger}\left(\boldsymbol{q}+\boldsymbol{G}^{\prime}\right)
\end{aligned}
$$

where $L^{2}$ is the $2 \mathrm{D}$ electron system area, $n_{\mathrm{F}}(x)=$ $\left\{\exp \left[(x-\mu) / k_{\mathrm{B}} T\right]+1\right\}^{-1}$ is the Fermi-Dirac occupation factor at temperature $T$ and chemical potential $\mu$, and, finally,

$$
\mathcal{M}_{\boldsymbol{k}, n, \nu ; \boldsymbol{k}^{\prime}, n^{\prime}, \nu}(\boldsymbol{q}+\boldsymbol{G}) \equiv\left\langle\boldsymbol{k}, n, \nu\left|e^{-i(\boldsymbol{q}+\boldsymbol{G}) \cdot \boldsymbol{r}}\right| \boldsymbol{k}^{\prime}, n^{\prime}, \nu\right\rangle,
$$

$|\boldsymbol{k}, n, \nu\rangle$ being the eigenstate of the non-interacting Hamiltonian for wave vector $\boldsymbol{k}$ in the $\mathrm{mBZ}$, band $n$, and principal valley $\nu$, and $\varepsilon_{\boldsymbol{k}, n, \nu}$ the corresponding eigenvalue.

Self-sustained oscillations of the total potential for vanishing external potential, which correspond to longitudinal collective electronic modes (i.e. plasmons), are found by solving $\operatorname{det}[\boldsymbol{\epsilon}(\boldsymbol{q}, \omega)]=0$. For each wave vector $\boldsymbol{q}$, the solution of this equation yields the complex angular

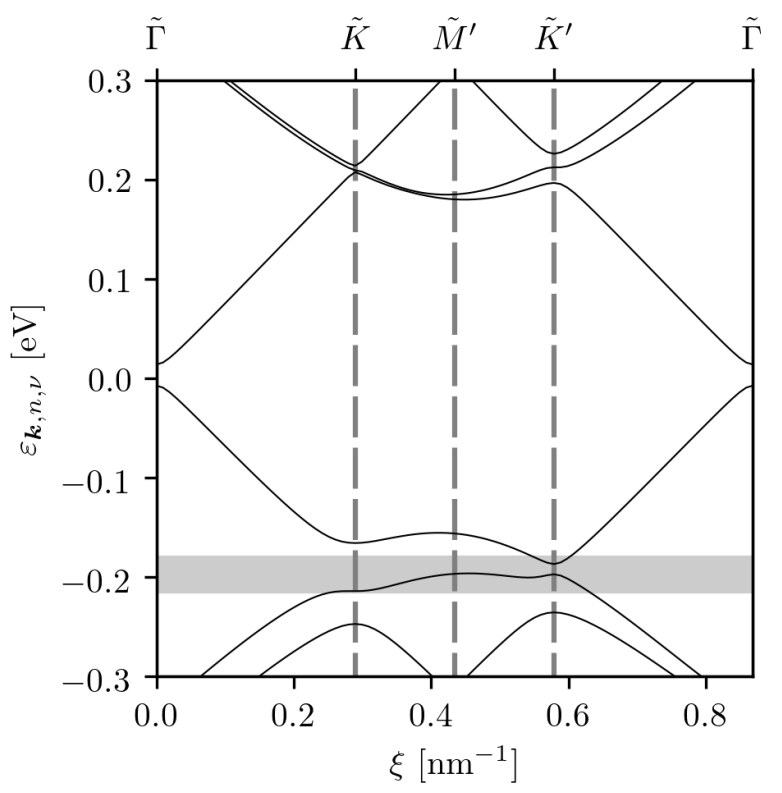

FIG. 1. Graphene-hBN superlattice minibands along the $\tilde{\Gamma}-\tilde{K}-\tilde{M}^{\prime}-\tilde{K}^{\prime}-\tilde{\Gamma}$ direction in the $\mathrm{mBZ}$, for the set of parameters given in Eq. 9, corresponding to vanishing twist angle $\theta=$ 0 . On the horizontal axis, the quantity $\xi$ indicates the total length along the path in the reciprocal space. The Fermi surfaces at the extrema of the shaded area are shown in Fig. 2.

frequency $\omega=\omega_{\mathrm{pl}}(\boldsymbol{q})$ of the plasmon mode. In the longwavelength limit, where $V_{\mathrm{sc}}(\boldsymbol{G}+\boldsymbol{q})$ decreases fast with $|\boldsymbol{G}|$, one can solve the simpler equation $\epsilon_{\mathbf{0}, \mathbf{0}}(\boldsymbol{q}, \omega)=0$. Moreover, assuming that the plasmon modes are longlived, one can solve for the roots with real angular frequency only, and then estimate the imaginary part. ${ }^{43}$

It is numerically more convenient, however, to calculate the so-called loss function

$$
L(\boldsymbol{q}, \omega)=-\Im m\left\{\left[\boldsymbol{\epsilon}^{-1}\right]_{\mathbf{0}, \mathbf{0}}(\boldsymbol{q}, \omega)\right\},
$$

which is proportional to the probability of exciting the $2 \mathrm{D}$ electron system by applying a perturbation with wave vector $\boldsymbol{q}$ and angular frequency $\omega$, and is directly measured e.g. via electron-energy-loss spectroscopy. ${ }^{44}$ More details on the calculation of the loss function and the eigenvectors of the moiré Hamiltonian can be found in Ref. 37. It is important to notice that, although the loss function depends only the $\boldsymbol{G}=\boldsymbol{G}^{\prime}=\mathbf{0}$ entry of the inverse dielectric matrix $\boldsymbol{\epsilon}^{-1}$, it is necessary to calculate the whole matrix $\boldsymbol{\epsilon}$ to perform the inversion.

\section{RESULTS}

In this Section, we present our numerical results for the loss function and associated plasmon spectrum.

The dispersion of the electronic energies in the $\mathrm{mBZ}$, dubbed "minibands," is shown in Fig. 1 along a path 


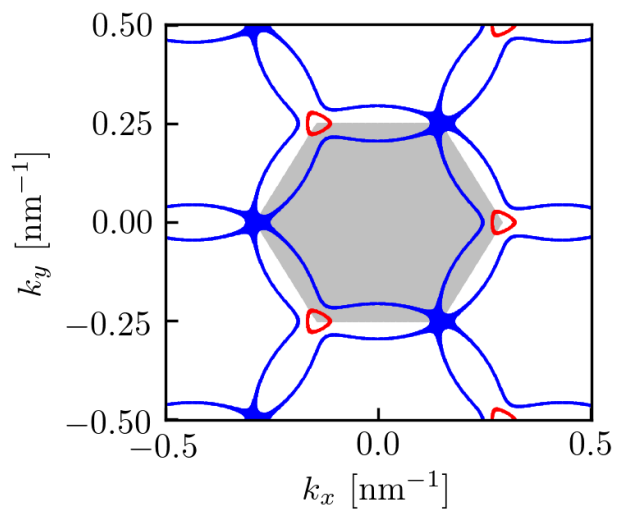

FIG. 2. (Color online) Fermi surfaces at the extrema of the shaded area shown in Fig. 1, for two Fermi energies $\varepsilon_{\mathrm{F}}=-180 \mathrm{meV}$ (red) and $-215 \mathrm{meV}$ (blue). The gray shaded area corresponds to the moiré superlattice Brillouin zone. The Fermi surfaces are periodically repeated in the reciprocal space for clarity.

in the mBZ around the $K$ valley of the original graphene Brillouin zone. Two gaps are clearly visible at the energy of the Dirac point $\varepsilon=0$ around the $\tilde{\Gamma}$ point of the mBZ and at $\varepsilon \simeq-200 \mathrm{meV}$ around the $\tilde{K}^{\prime}$ point. (Notice that, in the $K^{\prime}$ valley, the points $\tilde{K}$ and $\tilde{K}^{\prime}$ are exchanged.) The flatness of the second band below the Dirac point is noteworthy and clearly visible along the path between the $\tilde{K}$ and $\tilde{K}^{\prime}$ points. To better appreciate this band's flatness, in Fig. 2 we plot the Fermi surfaces for two different Fermi energies. The bottom of the first band below the Dirac point consists of one sharp minimum around the $\tilde{K}^{\prime}$ point, parabolic in shape but strongly anisotropic. The maximum of the second band, instead, is located at the $\tilde{M}$ point but, in a small energy range $\lesssim 5 \mathrm{meV}$, expands around the $\tilde{K}^{\prime}$ point, roughly in the shape of a three-blade propeller. Eventually, the tips of the bladelike shapes touch at the $\tilde{K}$ points and merge, yielding a familiar-looking but distorted hexagonal Fermi surface.

Fig. 3 contains the main results of this Section. It shows the loss function, at fixed wave vector, in a large chemical potential range. Above the Dirac point, we identify a single plasmon branch, almost unperturbed by the moiré potential with respect to the expression valid for massless Dirac fermions ${ }^{5}$

$$
\omega_{\mathrm{DF}}^{2}=\frac{2 \sqrt{\pi n(\mu)} v_{\mathrm{F}} e^{2}}{\hbar \bar{\epsilon}} q
$$

where $n(\mu)$ is the carrier density in the first conduction band, which depends on the chemical potential $\mu$. We emphasize that the spectral broadening of the plasmon branch, i.e. the width of the peak as a function of $\omega$, cannot be readily estimated from this density plot, because the extent of the monochrome shades has been truncated to improve the visibility of the less intense features. Around the Dirac point, the existence of the gap manifests as a forbidden band for the plasmon propagation, i.e. a region where plasmon branches are not

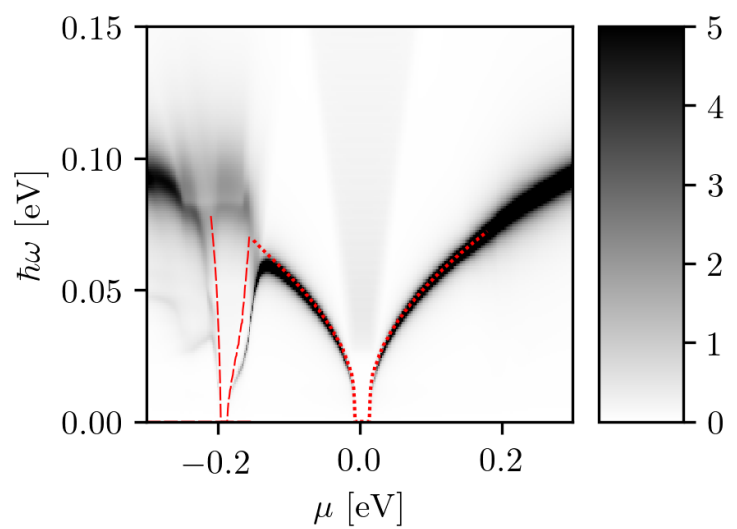

(a)

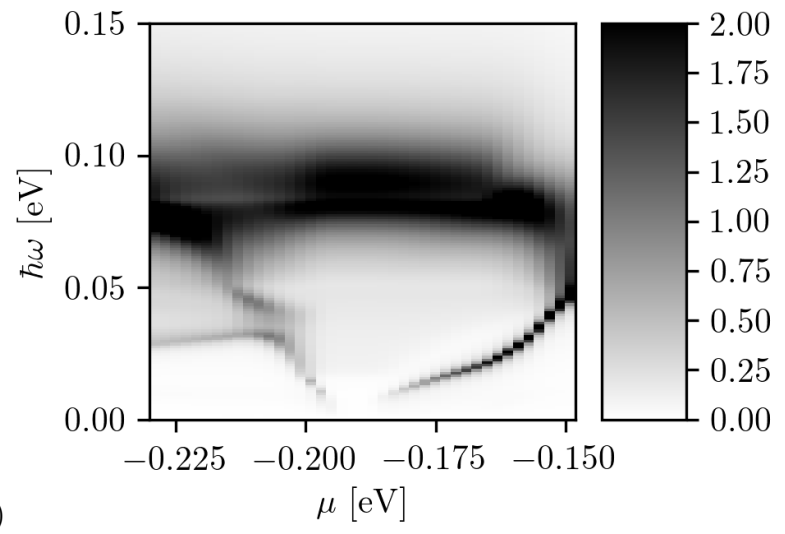

FIG. 3. (Color online) A 2D density plot of the RPA loss function $L(\boldsymbol{q}, \omega)$, as a function of the chemical potential $\mu$ and excitation energy $\hbar \omega$, for the parameters given in Eq. 9. The calculation is performed for a fixed wave vector of length $q=0.02 \mathrm{~nm}^{-1}$ along the $\tilde{M}-\tilde{\Gamma}$ direction. Panel (b) shows a magnification of (a) around the gap at $\mu \simeq-200 \mathrm{meV}$ (cfr. the minibands in Fig. 1). In (a) the red dotted line correspond to Eq. (16) and the red dashed line to Eq. (17). In both panels, and in the following figures as well, the range of the monochrome shades has been truncated to improve the visibility of the less intense features. For comparison, the graph of the loss function at fixed chemical potential is shown in Fig. 6(a).

supported. Moreover, inter-band transitions across the gap contribute a continuum of excitations which has the shape of an inverted, truncated cone.

The profile of the loss function below the Dirac point is dramatically different. As the chemical potential becomes more negative, the graphene's plasmon branch first grows in energy, following the expression (16) with $n(\mu)$ the hole density in the first valence band, and then bends abruptly to reach zero energy at the gap located around $\mu \simeq-200 \mathrm{meV}$. Below the gap, a plasmon branch rises again. This is an instance of the plasmon morphing phenomenon that was introduced and discussed in Ref. 37. As the band extrema above and below the gap are quadratic, the plasmon dispersion morphs following 


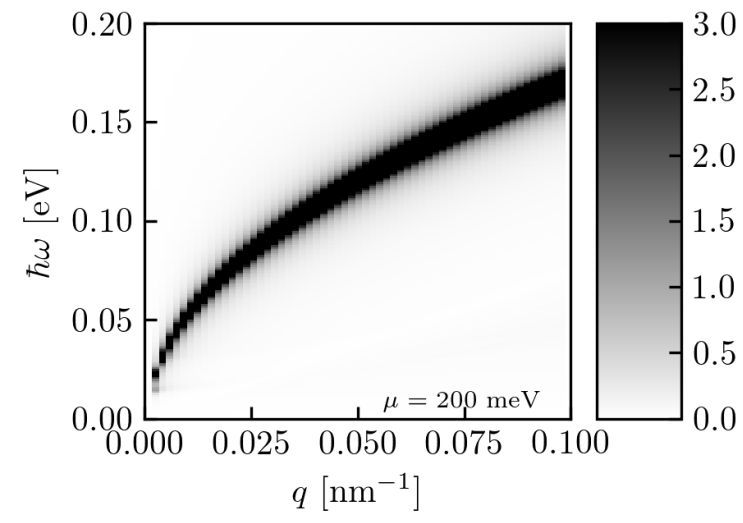

(a)

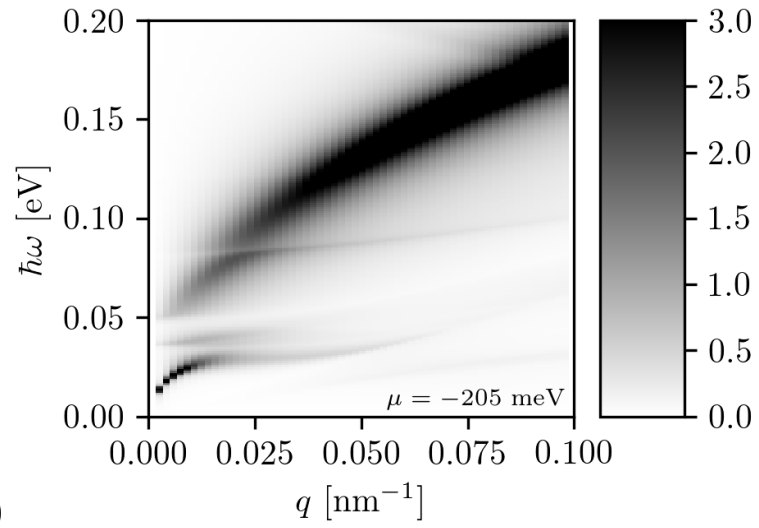

FIG. 4. A 2D density plot of the RPA loss function $L(\boldsymbol{q}, \omega)$, as a function of the wave vector length $q$ and the excitation energy $\hbar \omega$. The wave vector is taken along the $\tilde{M}-\tilde{\Gamma}$ direction. Results for positive $\mu=200 \mathrm{meV}$ and negative $\mu=-205 \mathrm{meV}$ chemical potentials are shown in (a) and (b), respectively.

the expression valid for a two-dimensional electron gas $^{39}$

$$
\omega_{2 \mathrm{DEG}}^{2}=\frac{2 \pi n(\mu) e^{2}}{m^{*} \bar{\epsilon}} q,
$$

where $m^{*} \simeq 0.01 m_{\mathrm{e}}$ is the carrier's effective mass, and $m_{\mathrm{e}}$ is the electron mass in vacuum. From the good qualitative agreement between the expression (17) and the profile of the loss function, it is clear that the main effect driving the plasmon morphing is the reduction (increase) of carrier density approaching (leaving) an extremal point of the band structure, i.e. the dependence of the Drude weight on the chemical potential, as discussed in detail in Ref. 37.

Across the gap, inter-band transitions contribute a thick continuum around $\hbar \omega \lesssim 10 \mathrm{meV}$. The location and extent of this continuum can be understood by looking at the Fermi surfaces just below and above the gap, shown in Fig. 2, which support a large number of electronic transitions with almost arbitrary wave vector in a restricted energy range. Most interestingly, below the gap and as the chemical potential changes, more than one plasmon branch and an apparent "avoided crossing" appear, suggesting that these branches correspond to coupled modes.
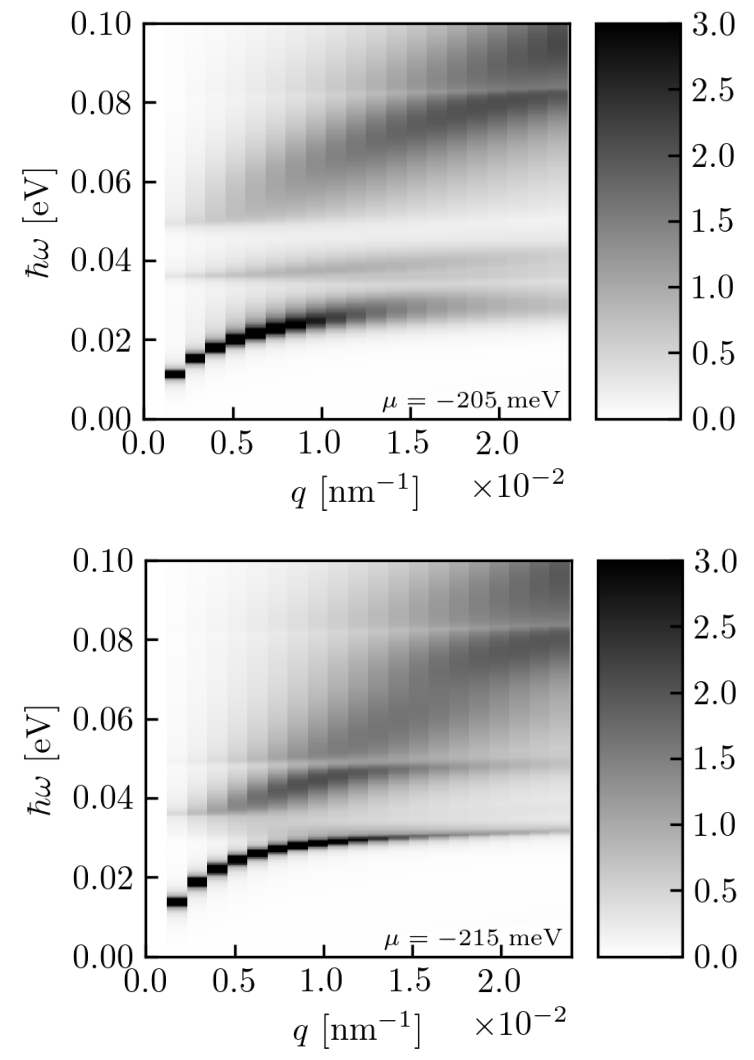

FIG. 5. As in Fig. 4, but in a smaller wave vector range around the origin of the reciprocal space. Results for two negative chemical potentials $\mu=-205 \mathrm{meV}$ and $-215 \mathrm{meV}$, slightly lower than the gap (cfr. the minibands in Fig. 1), are shown in (a) and (b), respectively.

To better appreciate the asymmetry of the plasmon spectrum above and below the Dirac point, in Fig. 4 we show the loss function as a function of the wave vector, for two values of the chemical potential. Above the Dirac point the plasmon's dispersion is almost unperturbed by the moiré potential. On the contrary, close to the gap at $\mu \simeq-200 \mathrm{meV}$, the low-energy dispersion $(\hbar \omega \lesssim 100 \mathrm{meV})$ is fractured into several branches with variable intensity, and recovers its almost unperturbed profile only at larger energies. Fig. 5 focuses on the lowenergy dispersion for two chemical potentials close to the gap. The dispersion is very similar, thus showing that the features discussed here are robust and do not depend on the specific value of the chemical potential. At very low energies $(\hbar \omega \lesssim 30 \mathrm{meV})$ a well-defined branch rises with $q$ and then flattens out, giving way to a continuum band of excitations, peaked around the unperturbed dispersion. The continuum band features a thin abrupt fracture around $\hbar \omega \simeq 50 \mathrm{meV}$, above which another continuum band appears.

To guide the interpretation of these spectral features, in Fig. 6(a) we juxtapose the loss function and the real and imaginary parts of the quantity $\epsilon_{\mathbf{0}, \mathbf{0}}(\boldsymbol{q}, \omega)$. Two sharp zeros of the real part are present where the loss 


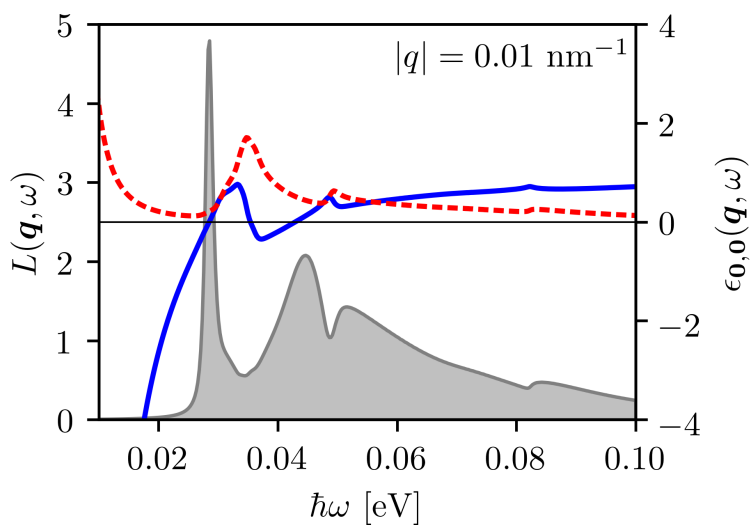

(a)

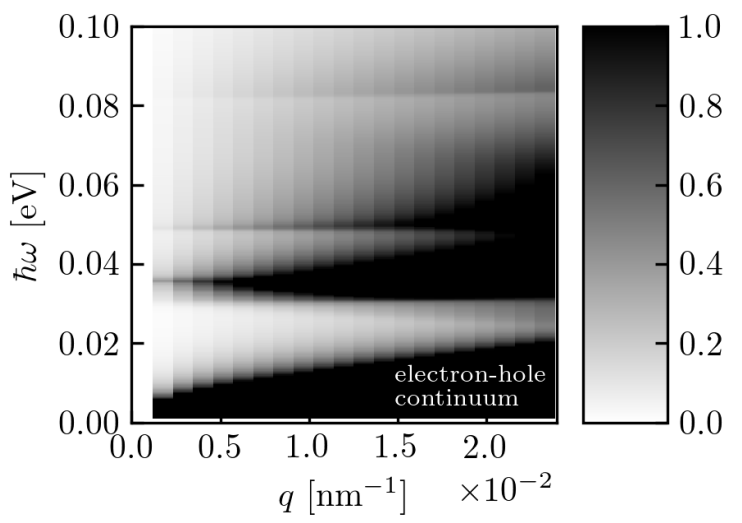

(b)

FIG. 6. (Color online) (a) The RPA loss function $L(\boldsymbol{q}, \omega)$ (shaded area), the real (solid line), and the imaginary (dashed line) part of the quantity $\epsilon_{\mathbf{0}, \mathbf{0}}(\boldsymbol{q}, \omega)$, as functions of the transition energy $\hbar \omega$, at fixed wave vector length $q=0.01 \mathrm{~nm}^{-1}$. The values of the loss (dielectric) function are reported on the left (right) vertical axis. (b) A 2D density plot of the imaginary part of the dielectric function, as a function of the wave vector length $q$ and the excitation energy $\hbar \omega$. In both panels, the wave vector is taken along the $\tilde{M}-\tilde{\Gamma}$ direction and the chemical potential is $\mu=-215 \mathrm{meV}$, as in Fig. 4(b).

function has its maxima. This shows that the maxima are indeed collective modes, and can be interpreted as branches of the plasmon dispersion. Between the maxima, a peak of the imaginary part of $\epsilon_{\mathbf{0}, \mathbf{0}}(\boldsymbol{q}, \omega)$ signals the existence of a continuum of electronic excitations, which separates the two branches. A more complete picture is obtained by looking at Fig. 6(b), which shows the imaginary part of $\epsilon_{\mathbf{0 , 0},}(\boldsymbol{q}, \omega)$, i.e. the electron-hole (e-h) continuum. In unperturbed graphene, at excitation energies which are small compared to the chemical potential, the e-h continuum consists of intra-band excitations below the "light-cone" $\omega=v_{\mathrm{F}} q$. Here, instead, it consists of a fractured domain, which includes bands extending horizontally with sharp bottom edges. Again, the origin of these bands can be qualitatively understood by referring to the large Fermi surfaces shown in Fig. 2. More precisely, elongated structures in the Fermi surface grant a support for e-h transitions with variable wave vector and constant energy, which coalesce into the horizontal bands of Fig. 6(b). In other words, inter-band transitions are possible across the shaded area shown in Fig. 1, in a large range of wave vectors. The dispersion of the plasmon modes cannot penetrate the intra-band e-h continuum, as is the case in unperturbed graphene, where the plasmon dispersion is tangent to the light-cone. Thus, the dispersion bends and follows the bottom edge of the e-h bands. Different branches effectively avoid each other, as if they were coupled modes, because they stem from the same set of electronic excitations.

A similar behavior of a dispersion relation is displayed by an electromagnetic mode at the interface between a metal and a dielectric. ${ }^{45}$ As the electromagnetic mode is coupled the the continuum of electronic transitions in the metal, its dispersion becomes sublinear and flattens out below the threshold for absorbtion due to surface plasmons modes. The resulting hybridized mode is known as surface plasmon-polariton. In the context of graphene bilayers and double-layers, modifications of the plasmon dispersion due to band nesting and inter-layer tunneling have been discussed in Refs. 46 and 47, respectively.

The results that we have presented above hold for nearly-aligned graphene and $\mathrm{hBN}$ layers, where $\theta \simeq 0$, because they crucially depend on the existence of the gap in the electronic dispersion at the $\tilde{K}^{\prime}$ point of the Brillouin zone around $\mu \simeq-200 \mathrm{meV}$. However, we have verified that the plasmon spectrum is asymmetric for a larger angle $\theta=2^{\circ}$ as well, although in a less dramatic fashion than displayed in Fig. 3. Since the exact angular dependence of the parameter $\Delta_{0}$ is not known analytically, and experimental reports of the gap magnitude are not in agreement ${ }^{22-26}$, we have used both $\Delta_{0}=0$ and $\Delta_{0}=10 \mathrm{meV}$ in the calculations.

\section{SUMMARY AND CONCLUSIONS}

In this work we have analyzed the plasmon spectrum of a heterostructure composed of two nearly-aligned layers of graphene and hexagonal boron nitride $(\mathrm{hBN})$. We have used a continuum-model effective Hamiltonian to obtain the dispersion relation of graphene's carriers in the heterostructure, which is different from that of isolated graphene because the $\mathrm{hBN}$ layer generates a periodic moiré potential for the carriers. We have discussed in detail the symmetry of the moiré potential and the relation between different formal representations of its functional form in real and sublattice space. The parameters of the moiré potential have been derived using a framework ${ }^{19}$ which combines symmetry considerations with input from $a b$ initio calculations. The electronic dispersion obtained with the continuum model consists of several minibands in the moiré superlattice Brillouin zones, centered at the $K$ and $K^{\prime}$ points of the Brillouin zone of pristine graphene, which shift in energy as the twist angle between the layers is varied. At vanishing twist angle between the layers, a gap is present about 200 meV below the Dirac point. We have calculated 
numerically the dielectric function and the loss function taking into account electronic transitions between minibands in the moiré Brillouin zones and electron-electron interactions at the level of the random phase approximation (RPA).

In conclusion, our calculations demonstrate a dramatic asymmetry of the plasmon dispersion at positive and negative chemical potential. This observation is potentially very relevant to establish the ideal working point of a graphene/hBN heterostructure as a two-dimensional platform for tunable, low-loss plasmonics. Moreover, around the gap below the Dirac point, the plasmon spectrum features several branches which appear as a result of a fractured electron-hole continuum due to the interband transitions between closely-spaced minibands with almost flat dispersion. Given the richness of the available band dispersion in graphene-based and, in general, in van der Waals heterostructures, our findings could be useful to guide further exploration of the non-trivial connection between the electronic and plasmonic dispersion in these systems.

\section{ACKNOWLEDGMENTS}

This work was supported by the Korean NRF through the grant number 2016R1A2B4010105 and by the 2018 sabbatical year research grant of the University of Seoul (J.J.) and the European Union's Horizon 2020 research and innovation programme under grant agreement No. 785219 - "GrapheneCore2" (A.T. and M.P.). Financial support from Consiglio Nazionale delle Ricerche (CNR) in the framework of the agreements on scientific collaborations between CNR and NRF (Korea) is also acknowledged in the initial stages of this work.
* andrea.tomadin@mac.com

† jeiljung@uos.ac.kr

1 K.S. Novoselov, A.K. Geim, S.V. Morozov, D. Jiang, Y. Zhang, S.V. Dubonos, I.V. Grigorieva, and A.A. Firsov, Science 306, 666 (2004).

2 K.S. Novoselov, D. Jiang, F. Schedin, T.J. Booth, V.V. Khotkevich, S.V. Morozov, and A.K. Geim, Proc. Natl. Acad. Sci. U.S.A. 102, 10451 (2005).

3 A.K. Geim and K.S. Novoselov, Nature Mater. 6, 183 (2007).

4 A.H. Castro Neto, F. Guinea, N.M.R. Peres, K.S. Novoselov, and A.K. Geim, Rev. Mod. Phys. 81, 109 (2009).

5 S. Das Sarma, S. Adam, E.H. Hwang, and E. Rossi, Rev. Mod. Phys. 83, 407 (2011).

6 C.R. Dean, A.F. Young, I. Meric, C. Lee, L. Wang, S. Sorgenfrei, K. Watanabe, T. Taniguchi, P. Kim, K.L. Shepard, and J. Hone, Nature Nanotechnol. 5, 722 (2010).

7 A.K. Geim and I.V. Grigorieva, Nature 499, 419 (2013).

8 J. Xue, J. Sanchez-Yamagishi, D. Bulmash, P. Jacquod, A. Deshpande, K. Watanabe, T. Taniguchi, P. Jarillo-Herrero, and B.J. LeRoy, Nature Mater. 10, 282 (2011).

9 X. Du, I. Skachko, F. Duerr, A. Luican, and E.Y. Andrei, Nature 462, 192 (2009).

10 K.I. Bolotin, F. Ghahari, M.D. Shulman, H.L. Stormer, and P. Kim, Nature 462, 196 (2009).

11 D.C. Elias, R.V. Gorbachev, A.S. Mayorov, S.V. Morozov, A.A. Zhukov, P. Blake, L.A. Ponomarenko, I.V. Grigorieva, K.S. Novoselov, F. Guinea, and A.K. Geim, Nature Phys. 7, 701 (2011).

12 R.V. Gorbachev, A.K. Geim, M.I. Katsnelson, K.S. Novoselov, T. Tudorovskiy, I.V. Grigorieva, A.H. MacDonald, S.V. Morozov, K. Watanabe, T. Taniguchi, and L.A. Ponomarenko, Nature Phys. 8, 896 (2012).

13 M. Yankowitz, J. Xue, D. Cormode, J.D. SanchezYamagishi, K. Watanabe, T. Taniguchi, P. Jarillo-Herrero, P. Jacquod, and B.J. LeRoy, Nat. Phys. 8, 382 (2012).

14 M. Kindermann, B. Uchoa, and D.L. Miller, Phys. Rev. B 86, 115415 (2012)
15 F. Amet, J.R. Williams, K. Watanabe, T. Taniguchi, and D. Goldhaber-Gordon, Phys. Rev. Lett. 110, 216601 (2012).

16 J.R. Wallbank, A.A. Patel, M. Mucha-Kruczyński, A.K. Geim, and V.I. Fal'ko, Phys. Rev. B 87, 245408 (2013).

17 J. Jung, A.M. DaSilva, A.H. MacDonald, and S. Adam, Nature Commun. 6, 6308 (2015).

18 A.M. DaSilva, J. Jung, S. Adam, and A.H. MacDonald, Phys. Rev. B 91, 245422 (2015).

19 J. Jung, E. Laksono, A.M. DaSilva, A.H. MacDonald, M. Mucha-Kruczyński, and S. Adam, Phys. Rev. B 96, 085442 (2017).

20 C.R. Dean, L. Wang, P. Maher, C. Forsythe, F. Ghahari, Y. Gao, J. Katoch, M. Ishigami, P. Moon, M. Koshino, T. Taniguchi, K. Watanabe, K.L. Shepard, J. Hone, and P. Kim, Nature 497, 598 (2013).

21 L.A. Ponomarenko, R.V. Gorbachev, G.L. Yu, D.C. Elias, R. Jalil, A.A. Patel, A. Mishchenko, A.S. Mayorov, C.R. Woods, J.R. Wallbank, M. Mucha-Kruczynski, B.A. Piot, M. Potemski, I.V. Grigorieva, K.S. Novoselov, F. Guinea, V.I. Fal'ko, and A.K. Geim, Nature 497, 594 (2013).

22 B. Hunt, J.D. Sanchez-Yamagishi, A.F. Young, M. Yankowitz, B.J. LeRoy, K. Watanabe, T. Taniguchi, P. Moon, M. Koshino, P. Jarillo-Herrero, and R.C. Ashoori, Science 340, 1427 (2013).

23 Z.-G. Chen, Z. Shi, W. Yang, X. Lu, Y. Lai, H. Yan, F. Wang, G. Zhang and Z. Li, Nature Commun. 5, 4461 (2014).

24 E. Wang, X. Lu, S. Ding, W. Yao, M. Yan, G. Wan, K. Deng, S. Wang, G. Chen, L. Ma, J. Jung, A.V. Fedorov, Y. Zhang, G. Zhang, and S. Zhou, Nature Phys. 12, 1111 (2016).

25 C.R. Woods, L. Britnell, A. Eckmann, R.S. Ma, J.C. Lu, H.M. Guo, X. Lin, G.L. Yu, Y. Cao, R.V. Gorbachev, A.V. Kretinin, J. Park, L.A. Ponomarenko, M.I. Katsnelson, Y.N. Gornostyrev, K. Watanabe, T. Taniguchi, C. Casiraghi, H.-J. Gao, A.K. Geim, and K.S. Novoselov, Nature Phys. 10, 451 (2014).

26 H. Kim, N. Leconte, B. L. Chittari, K. Watanabe, T. Taniguchi, A. H. MacDonald, J. Jung, and S. Jung, 
arXiv:1808.06633 (2018).

27 P. San-Jose, A. Gutiérrez-Rubio, M. Sturla, and F. Guinea, Phys. Rev. B 90, 75428 (2014).

${ }^{28}$ F. Bonaccorso, Z. Sun, T. Hasan, and A. Ferrari, Nature Photon. 4, 611 (2010).

29 A. Grigorenko, M. Polini, and K. Novoselov, Nature Photon. 6, 749 (2012)

30 A. Woessner, M.B. Lundeberg, Y. Gao, A. Principi, P. Alonso-González, M. Carrega, K. Watanabe, T. Taniguchi, G. Vignale, M. Polini, J. Hone, R. Hillenbrand, and F.H.L. Koppens, Nature Mater. 14, 421 (2015).

31 Z. Fei, A.S. Rodin, G.O. Andreev, W. Bao, A.S. McLeod, M. Wagner, L.M. Zhang, Z. Zhao, M. Thiemens, G. Dominguez, M.M. Fogler, A.H. Castro Neto, C.N. Lau, F. Keilmann, and D.N. Basov, Nature 487, 82 (2012).

32 J. Chen, M. Badioli, P. Alonso-González, S. Thongrattanasiri, F. Huth, J. Osmond, M. Spasenović, A. Centeno, A. Pesquera, P. Godignon, A. Zurutuza Elorza, N. Camara, F.J. García de Abajo, R. Hillenbrand, and F.H.L. Koppens, Nature 487, 77 (2012).

${ }^{33}$ G.X. Ni, H. Wang, J.S. Wu, Z. Fei, M.D. Goldflam, F. Keilmann, B. Özyilmaz, A.H. Castro Neto, X.M. Xie, M.M. Fogler, and D.N. Basov, Nature Mater. 14, 1217 (2015).

${ }^{34}$ G.X. Ni, A.S. McLeod, Z. Sun, L. Wang, L. Xiong, K.W. Post, S.S. Sunku, B.-Y. Jiang, J. Hone, C.R. Dean, M.M. Fogler, and D.N. Basov, Nature 557, 530 (2018).

35 D.N. Basov, M.M. Fogler, and F.J. García de Abajo, Science 354, aag1992 (2016).
36 T. Low, A. Chaves, J. Caldwell, A. Kumar, N. Fang, P. Avouris, T. Heinz, F. Guinea, L. Martin-Moreno, and F. Koppens, Nature Mater. 16, 182 (2017).

37 A. Tomadin, F. Guinea, and M. Polini, Phys. Rev. B 90, 161406(R) (2014).

38 J. Jung, A. Raoux, Z. Qiao, and A.H. MacDonald, Phys. Rev. B 89, 205414 (2014).

39 G.F. Giuliani and G. Vignale, Quantum Theory of the Electron Liquid (Cambridge University Press, Cambridge, 2005).

40 In Ref. 19, the notation $\varphi_{A B}=\pi / 6-\phi_{x y}$ was used.

41 J.R. Wallbank, M. Mucha-Kruczyński, X. Chen, and V.I. Fal'ko, Ann. Phys. (Berlin) 527, 359 (2015).

42 In this work, we neglect Hartree corrections to the Lindhard function, which in general are necessary to formulate the RPA of inhomogeneous systems.

43 A.L. Fetter and J.D. Walecka, Quantum Theory of ManyParticle Systems (Dover Publications, 2003).

${ }^{44}$ R.F. Egerton, Rep. Prog. Phys. 72, 016502 (2009).

45 S.A. Maier, Plasmonics: Fundamentals and Applications (Springer, 2007).

46 T. Low, F. Guinea, H. Yan, F. Xia, and P. Avouris, Phys. Rev. Lett. 112, 116801 (2014).

47 D. Svintsov, Zh. Devizorova, T. Otsuji, and V. Ryzhii, Phys. Rev. B 94, 115301 (2016). 